\title{
AI-aided design of novel targeted covalent inhibitors against SARS-CoV-2
}

\author{
Bowen Tang ${ }^{1,2}$, Fengming $\mathrm{He}^{l}$, Dongpeng Liu ${ }^{2}$, Meijuan Fang ${ }^{l}$, Zhen $W u^{l, *}$ and Dong $X u^{2, *}$
}

${ }^{1}$ Fujian Provincial Key Laboratory of Innovative Drug Target Research, School of Pharmaceutical Sciences, Xiamen University, Xiamen 361000, China

${ }^{2}$ Department of Electrical Engineering and Computer Science, Informatics Institute, and Christopher S. Bond Life Sciences Center, University of Missouri, Columbia, MO 65211, USA

*Correspondence: wuzhen@xmu.edu.cn, xudong@missouri.edu

\begin{abstract}
The focused drug repurposing of known approved drugs (such as lopinavir/ritonavir) has been reported failed for curing SARS-CoV-2 infected patients. It is urgent to generate new chemical entities against this virus. As a key enzyme in the life-cycle of coronavirus, the $3 \mathrm{C}$-like main protease $\left(3 \mathrm{CL}^{\text {pro }}\right.$ or $\left.\mathrm{M}^{\text {pro }}\right)$ is the most attractive for antiviral drug design. Based on a recently solved structure (PDB ID: 6LU7), we developed a novel advanced deep Q-learning network with the fragment-based drug design (ADQN-FBDD) for generating potential lead compounds targeting SARS-CoV$23 \mathrm{CL}^{\text {pro }}$. We obtained a series of derivatives from those lead compounds by our structure-based optimization policy (SBOP). All the 47 lead compounds directly from our AI-model and related derivatives based on SBOP are accessible in our molecular library at https://github.com/tbwxmu/2019-nCov. These compounds can be used as potential candidates for researchers in their development of drugs against SARS-CoV-
\end{abstract} 2.

\section{Introduction}

The emerging coronavirus SARS-CoV-2 has caused an outbreak of coronavirus 
disease (COVID-19) worldwide. ${ }^{1}$ As of March 2, 2020, more than 90,000 people have been infected by SARS-CoV-2 and more than 3000 people have been reported dead according to Johns Hopkins Coronavirus map tracker. ${ }^{2}$ The numbers of infection and death are still increasing. To face the considerable threat of SARS-CoV-2, it is urgent to develop new inhibitors or drugs against this deadly virus. Unfortunately, since the outbreak of severe acute respiratory syndrome (SARS) eighteen years ago, there has been no approved treatment against the SARS coronavirus (SARS-CoV), ${ }^{3}$ which is similar to SARS-CoV-2. Repurposing potential drugs such as lopinavir and ritonavir also failed to SARS-CoV-2 injected patients. ${ }^{4}$ Structure-based antiviral drug design with a new artificial intelligence algorithm may represent a more helpful approach to get the SARS-CoV-2 targeted inhibitors or drugs. Thanks to the prompt efforts of many researchers, we have several pieces of important information about this vital virus genome and protein structures. We now know that the non-structural protein 5 (Nsp5) is the main protease $\left(\mathrm{M}^{\text {pro }}\right)$ of SARS-CoV-2 and it is a cysteine protease, which also been called "3C-like protease" (3CL pro). Moreover, we know that the 3D structure of $3 \mathrm{CL}^{\text {pro }}$ is very similar to SARS-CoV with a sequence identity of $>96 \%$ and $3 \mathrm{D}$ structure superposition $\mathrm{RMSD}_{\mathrm{C} \alpha}$ of $0.44 \AA$ as shown in Figures $\mathrm{S} 1$ and $\mathrm{S} 2$.

$3 \mathrm{CL}^{\text {pro }}$ has been reported as an attractive target for developing anti-coronaviral drugs: 1) this protease is highly conserved in both sequences and $3 \mathrm{D}$ structures; ${ }^{5}$ 2) $3 \mathrm{CL}^{\text {pro }}$ is a key enzyme for related virus (including SARS and SARS-CoV-2) replication; 3) it only exists in the virus, not in humans. Developing specific antiviral drugs targeting $3 \mathrm{CL}^{\text {pro }}$ of the specific virus has shown significant success; for example, both approved drugs lopinavir and ritonavir can completely occupy the substrate-binding site of $3 \mathrm{CL}^{\text {pro }}$ to break down the replication of human immunodeficiency virus (HIV). However, due to the large difference between HIV and SARS-CoV-2 3CL pro lopinavir and ritonavir were validated ineffective for inhibiting SARS-Cov-2. ${ }^{4}$ On the other hand, the substrate-binding site of $3 \mathrm{CL}^{\text {pro }}$ is almost the same between the SARS-CoV-2 and SARS as Figure S3 presents. The developed potential inhibitors and drug-design experience targeting SARS-3CL ${ }^{\text {pro }}$ may also be applicable to SARS-CoV-2. For 
example, the recently solved structure of SARS-CoV-2 3CL pro (PDB ID: 6LU7) indicates that the developed inhibitor $\mathrm{N} 3,{ }^{6}$ which is a covalent inhibitor derived from non-covalent inhibitors against SARS can also bind SARS-CoV-2 3CL pro with a similar binding conformation (Figure S4).

All the above available information paved a way to design new targeted covalent inhibitors (TCI) ${ }^{7}$ against SARS-CoV-2. A successful TCI against $3 \mathrm{CL}^{\text {pro }}$ must first be able to fit in the binding site of $3 \mathrm{CL}^{\text {pro }}$ with an appropriate pose that keeps its reactive groups close enough to the Cys145, which then undergoes a chemical step (nucleophilic attack by Cys145) leading to the formation of a stable covalent bond as presented in the scheme below:

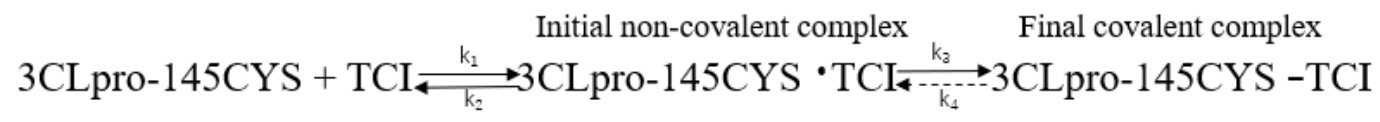

TCIs usually have a longer target residence time than the relative non-covalent inhibitors in theory given the following: 1) For the inhibition, $\mathrm{k}_{1}$ must be larger than $\mathrm{k}_{2}$, and thus the non-covalent binding is determined by the equilibrium constant $\mathrm{k}_{1} / \mathrm{k}_{2} ; 2$ ) TCIs have the chemical reaction step with the target, where usually $\mathrm{k}_{3}>>\mathrm{k}_{4}$; and 3 ) for TCIs, the binding process is controlled by $\mathrm{k}_{1} \mathrm{k}_{3} /\left(\mathrm{k}_{2} \mathrm{k}_{4}\right)$, which is bigger than $\mathrm{k}_{1} / \mathrm{k}_{2}$ in non-covalent inhibitors. In some extreme cases $\mathrm{k}_{4}=0$, and hence, these irreversible inhibitors covalently bind the target until the target disappears. ${ }^{7,8}$

Considering the inhibitors of SARS 3CL pro may be also bio-active to SARS-CoV-2, we have created a molecular library including all the reported SARS-3CL pro inhibitors (284 molecules $)^{9}$ 10, 11, 12, 13, 14, 15, 16, 17, 18, 19, 20, 21, 22, 23, 24, 25, 26, 27, 28, 29 and we will also add new validated molecular structures into this library with our research progresses. To date, there are no clinically approved vaccines or drugs specifically targeting SARS-CoV-2. Thus, with the hope to discover novel candidate drugs targeting SARS-CoV-2, we combine artificial intelligence (AI) with the structure-based drug design (SBDD) to accelerate generating potential lead compounds and design TCIs. 


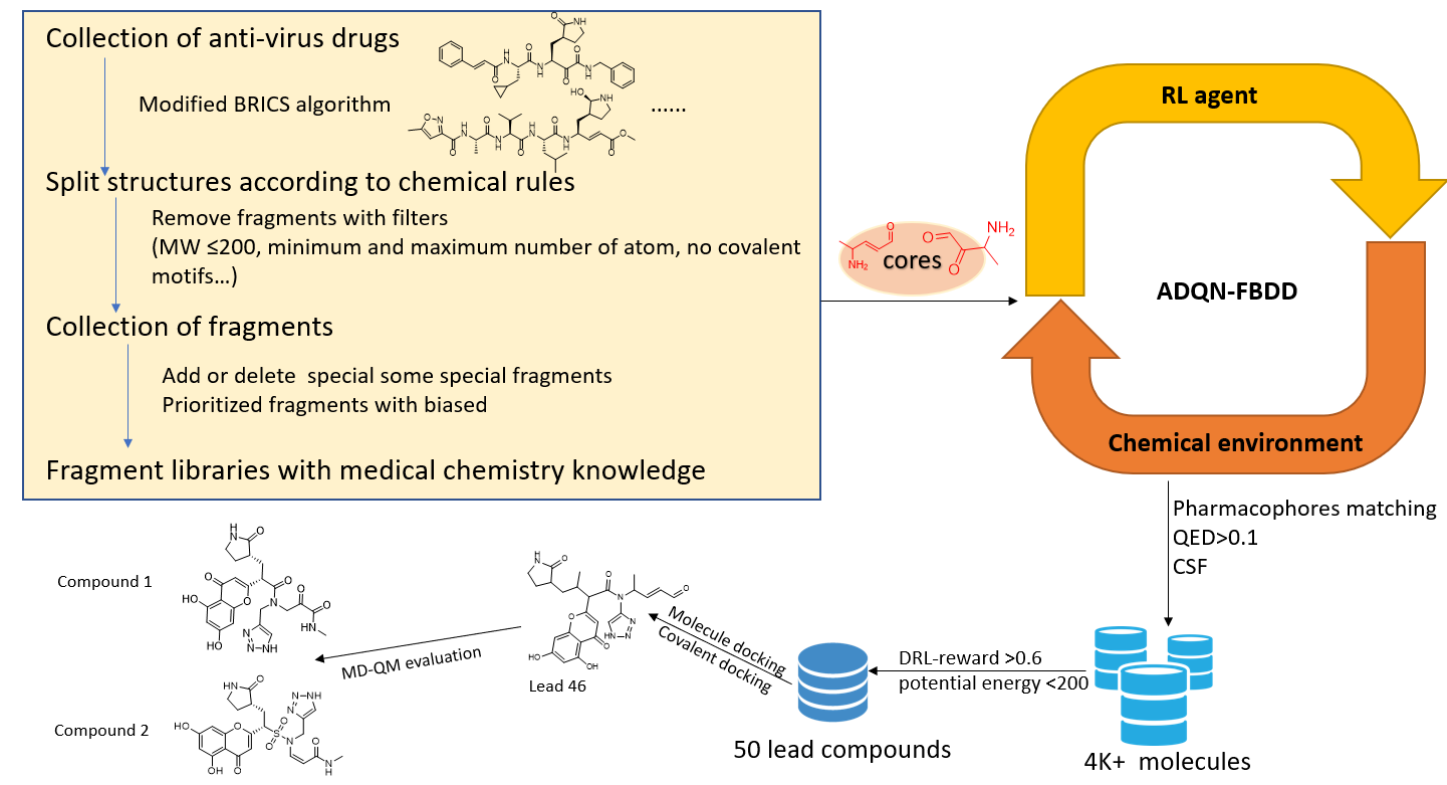

Figure 1. Flowchart for our SARS-CoV-2 3CL pro lead compounds development.

AI, especially deep learning, has been applied in predicting molecular properties ${ }^{30,31,}$ 32, 33 and designing novel molecules ${ }^{34}$. Unlike earlier deep-learning molecular design by adding single atom one at a time ${ }^{34,35}$, our approach explores new molecules by adding a meaningful molecular fragment one by one, which is not only computationally more efficient but also chemically more reasonable. To make our AI model work well, the first step is to prepare the molecular fragment library as shown in Figure 1. We used our collected SARS-CoV 3CL ${ }^{\text {pro }}$ inhibitors (284 molecules) as the initial molecule database targeting SARS-CoV-2 3CL pro. Then, we split this set of molecules into fragments with a molecule weight no more than 200 daltons. Both of the collected inhibitors and the fragments are supplied in https://github.com/tbwxmu/2019-nCov. Then we applied an advanced deep Q-learning network with the fragment-based drug design (ADQN-FBDD) for generating potential lead compounds. Noted, if researchers have enough experience and internal lead compounds or biased fragments, they can inject all such information into ADQN-FBDD by manually adding the lead compounds and biased fragments to the corresponding files. By using the same fragments directly from existing bioactivate molecules, our ADQN-FBDD agent can easily access the potential chemical space focused on $3 \mathrm{CL}^{\text {pro }}$ of SARS-CoV-2. 
After ADQN-FBDD automatically generated novel compounds targeting this new virus $3 \mathrm{CL}^{\text {pro }}$, we obtained a covalent lead compound library with 4,922 unique valid structures. In total, 47 of these compounds were selected with high scores from our AImodel's reward function. Then these molecules were further evaluated by docking and covalent docking studies. The lead compound \#46 with a high covalent docking score attracts our attention, which also has a low difference between non-covalent and covalent docking pose among the 47 lead compounds. After carefully checking the lead \#46's interaction mode with $3 \mathrm{CL}^{\text {pro }}$, we believe there is still much space to optimize lead it. Then we designed a series of derivatives from compound \#46 based on our chemical biology knowledge and the structure-based optimization policy. All the generated molecular structures are published in our code library https://github.com/tbwxmu/2019-nCov. We encourage researchers who are interested in finding a potential treatment for this viral infection to synthesize and evaluate some of these molecules for treating COVID-19.

\section{Results}

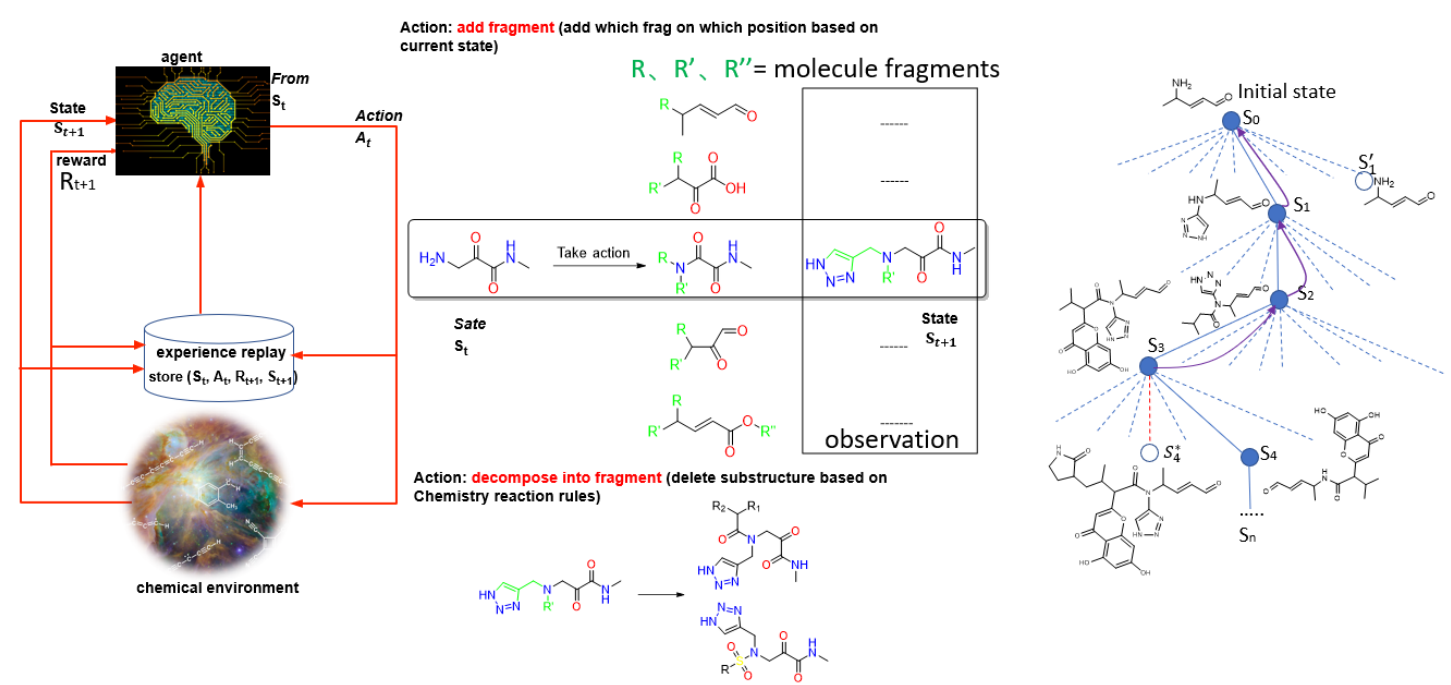

Figure 2. A: Framework of ADQN-FBDD. B: Examples for explaining the fragment-based actions. C:

The solid lines represent taken actions including the addition/deletion of a different fragment or no modification during each step. The dashed lines represent actions that our reinforcement learning agent considered but did not make. After the first three actions to $S_{1}, S_{2}$, and $S_{3}$, the fourth action to $S_{4}$ was an exploratory action, meaning that it was taken even though another sibling action, the one colored in red 
dashed line leading to $s_{4}^{*}$, was ranked higher. Exploratory action does not result in any learning, but each of our other actions does, resulting in updates as suggested by the purple curved arrow in which estimated values are moved up the tree from later nodes to earlier ones.

Integrating the double dueling deep Q learning with fixed q-targets and prioritized experience replay enables our agent ADQN stable and efficient during learning from the chemical environment. Combining the state-of-the-art AI algorithm with the idea of FBDD as presented in Figure 2, ADQN-FBDD is flexible and efficient to access the focused chemical space targeting the SARS-CoV-2 3CL pro. Based on the configurations targeting the SARS-CoV-2 3CL pro, ADQN-FBDD generated a potential lead library containing 4,922 unique molecular structures (Supplementary dataset in https://github.com/tbwxmu/2019-nCov). To narrow our focus to a smaller set of molecules for analysis, we elaborately defined filter rules (QED $>0.1$ and DRLreward $>=0.6$ ) and the detailed information of the rules can be found in the Methods section. And then 47 unique molecules (Table S1) were kept for the next non-covalent docking and covalent-docking evaluation. These 47 virtual leads display suitable 3Dcomplexity with common characteristics of peptidomimetics and protein-protein interaction (PPI) inhibitors. They are mainly ranked by covalent-docking scores, considering covalent-docking also contains the scores of non-covalent docking. ${ }^{36}$ And we also paid attention on the RMSD difference between the covalent binding and noncovalent binding poses based on all heavy atoms. Finally, we selected the lead molecule \#46 as displayed in Figure 3 with both a good covalent docking score and a small RMSD value (Table S1). We further optimized it and get a series of derivatives based on the SBDD approach.

Molecule \#46 was ranked number 1 based on the covalent docking score and its interaction model with the binding site was carefully checked as shown in Figure 3. Although compound \#46 has the best covalent docking score, it has the alerting group aldehyde. Considering there is still much space for compound \#46 to fill in the S1' subsite and $\alpha$-ketoamides may be good to fit the oxyanion hole (Figure $4 \mathrm{~A}$ ) of $3 \mathrm{CL}^{\text {pro }}, 3$ 
we replaced the aldehyde by formamide and also replaced the 1,4 Michael acceptors by alpha-ketoamides. Thus, we optimized compound \#46 to compound 46-14-1 (Figure 4 $\mathrm{A}$ and $\mathrm{B})$.

A

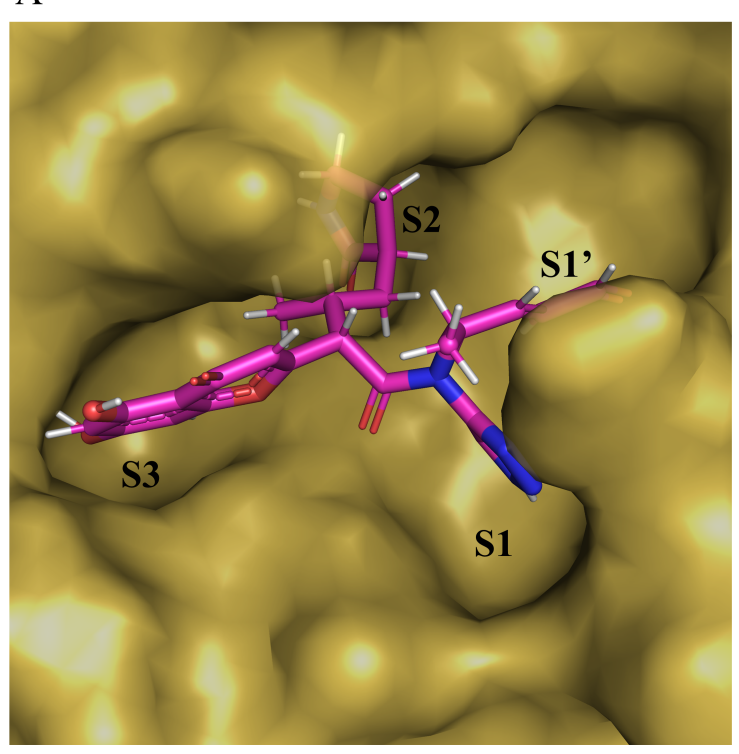

$\mathrm{C}$

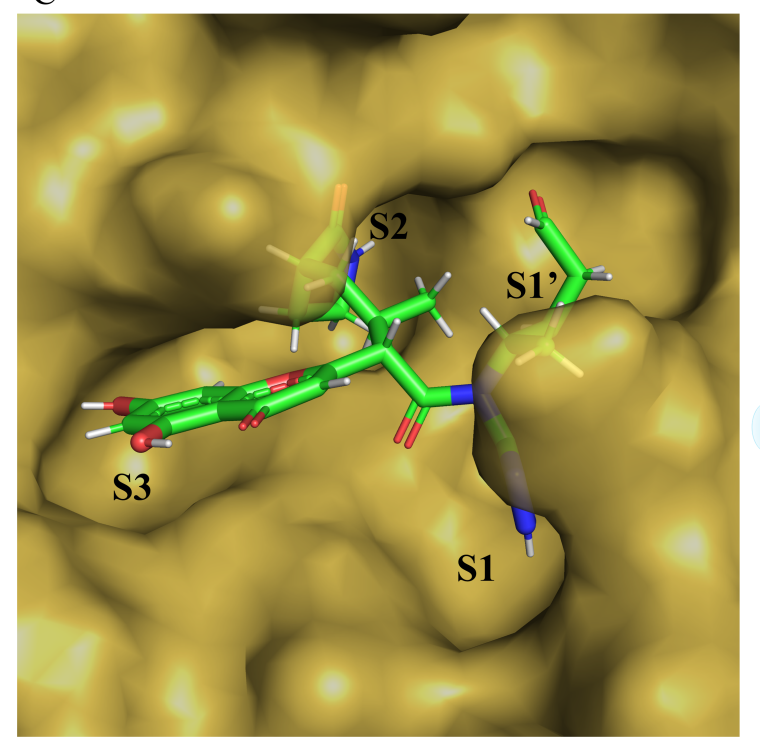

$\mathrm{B}$

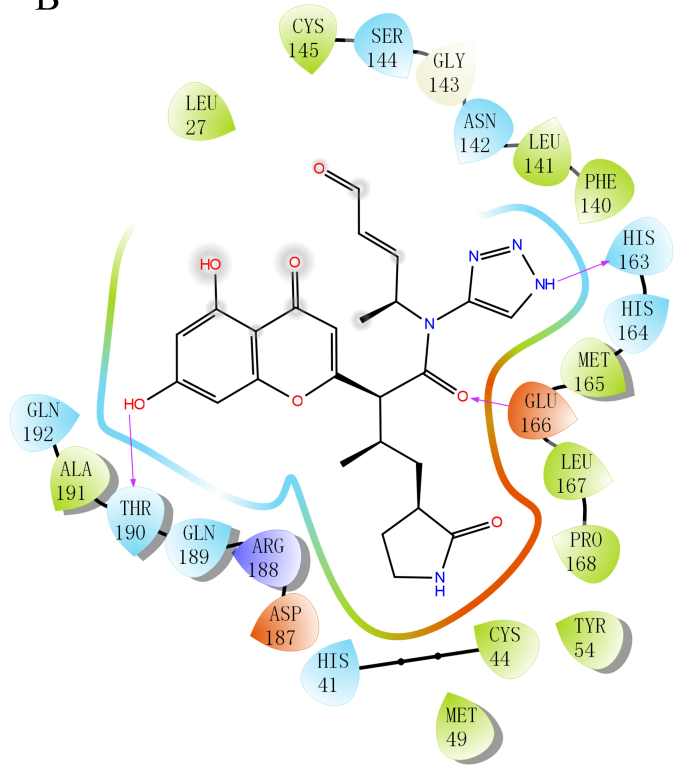

$\mathrm{D}$

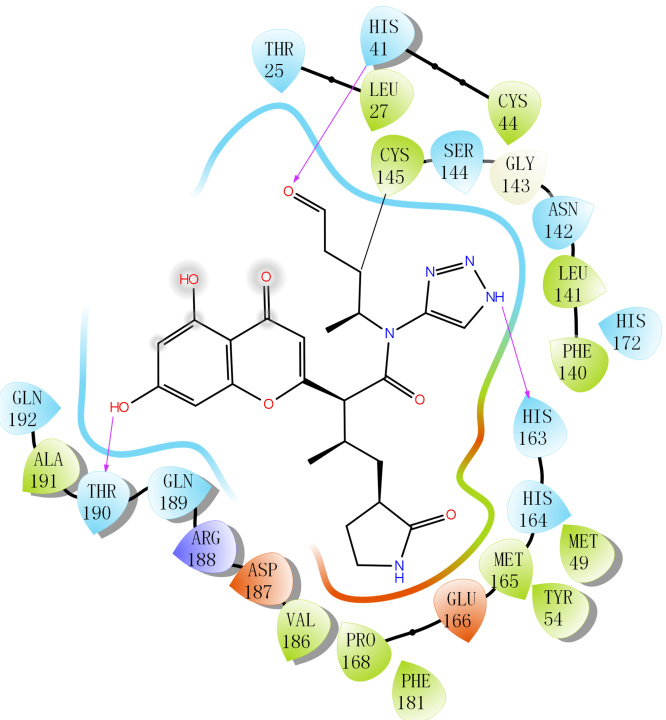

Figure 3. Lead compound \#46 generated by AI. A: Non-covalent molecular docking model of SARS-CoV-2 $3 \mathrm{CL}^{\text {pro }}$ (brown surface) with the bound lead compound \#46 (magenta sticks). The triazole ring binds to the $\mathrm{S} 1$ subsite of the catalytic active center, the covalent fragment of $\alpha, \beta$ unsaturated aldehyde binds to $\mathrm{S} 1$ ' subsite, $\beta$-lactam ring binds to $\mathrm{S} 2$ subsite and 5,7-dihydroxy chromone binds to $\mathrm{S} 3$ subsite. B: A two-dimensional (2D) view of non-bonding interaction of lead compound \#46 in complex with 3CL protease based on non-covalent docking. The triazole ring, i.e., keto-amide, phenolic hydroxy forms hydrogen bond (H-bond) with His163, Glu166, and Thr190, respectively. C: Covalent docking model between compound \#46 (green sticks) and 3CL protease (brown surface) exhibits a similar docking model as non-covalent docking. D: A 2D view of ligand 
interaction between compound \#46 and protease under covalent docking. The triazole ring forms an H-bond with His 163; the fragment of $\alpha, \beta$-unsaturated aldehyde forms a covalent bond with Cys145, i.e., the key residue in the catalytic center of the protease, resulting in covalent inhibition; aldehyde carbonyl forms an H-bond with His41; and besides, the hydroxyl of chromone at position 7 forms an H-bond with Thr190.

The non-bonding interaction between compound 46-14-1 and SARS-CoV-2 $3 \mathrm{CL}^{\text {pro }}$ is mainly hydrogen bond. The carbonyl group of covalent scaffold $\alpha$-ketoamide forms $\mathrm{H}$ bonds with Leu141 and Gly143 as a hydrogen acceptor or a hydrogen donor. The hydrogen on the nitrogen atom of the triazole ring forms an H-bond with Glu166, while Glu166 also forms an H-bond with the carbonyl group on the main chain. The oxygen in the $\beta$-lactam ring forms an H-bond with His41. In order to enhance the polarity of the compounds, sulfonic groups were introduced to replace the ketone carbonyl groups on the main chain and sulfonamides 46-14-2 were obtained. The covalent docking model of compound 46-14-2 with SARS-CoV-2 3CL pro is shown in Figure $4 \mathrm{C}$ and D.

In order to make the compound 46-14-2 fit the active pocket with a higher affinity, we added a carbon atom to the sulfonic acid group of the original molecule, extending the carbon chain to increase the flexibility of the molecule, and obtained another optimized compound 46-14-3. The mode of covalent docking with SARS-CoV-2 $3 \mathrm{CL}^{\text {pro }}$ is shown in Figure 5. Due to the introduction of carbon atoms and the enhancement of molecular flexibility, the $\beta$-lactam ring can be inserted deeper into the S2 pocket and the other fragments of the compound can better adapt to the S1, S1' and S3 subsites. The $\alpha$ carbonyl carbon on the $\alpha$-ketoamide of compound 46-14-3 forms a covalent bond with the key residue Cys145 on the protease, but the main non-bond interaction is still hydrogen bond (indicated by a yellow dash). The triazole ring mainly forms H-bonds with Phe140 and Glu166 residues in the S1 pocket; $\alpha$-ketoamide covalent binding fragment mainly forms H-bonds with key amino acid residues Cys145, Gly143 and Ser144 in the S1' subsite, which forms an oxyanion hole in the red circle in Figure 9; the $\beta$-lactam side chain mainly forms H-bonds with residues Tyr54 and AsS187 in the 
S2 pocket; the chromone scaffold mainly forms H-bonds with key residues Thr190 and Gln192 in the S3 pocket. In addition, the oxygen on the sulfonyl group of the main chain forms an H-bond with Glu166 in the S1 pocket.

A

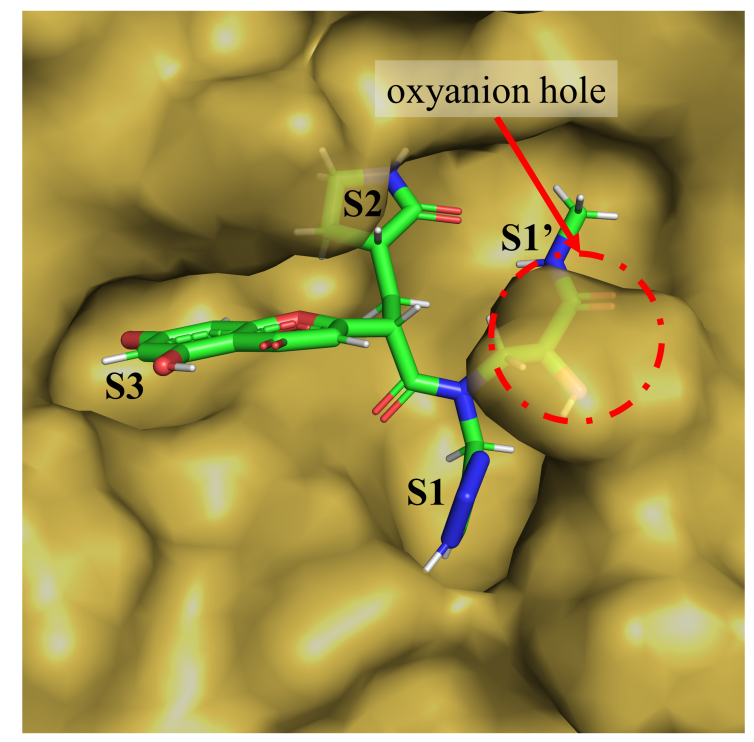

$\mathrm{C}$

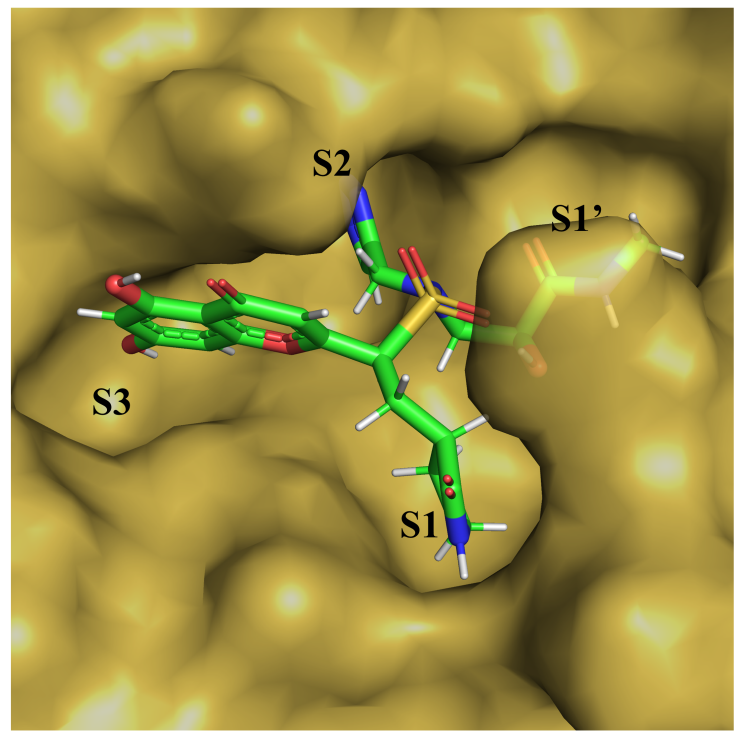

$\mathrm{B}$

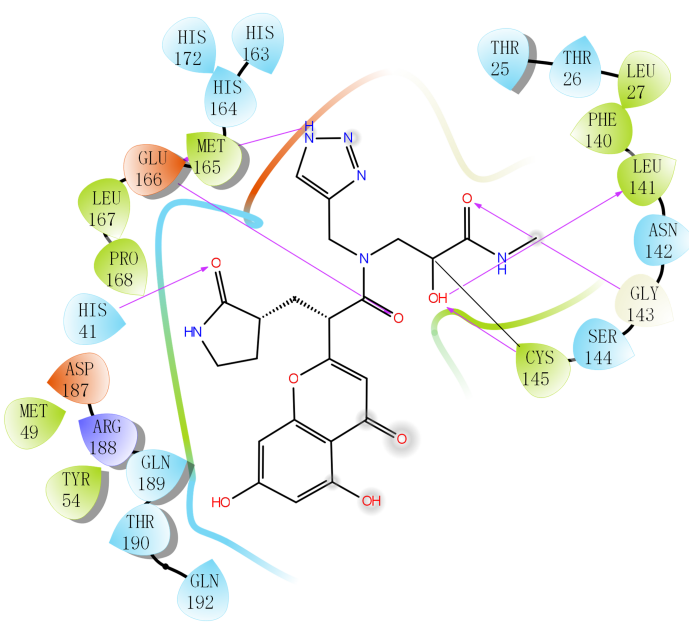

$\mathrm{D}$

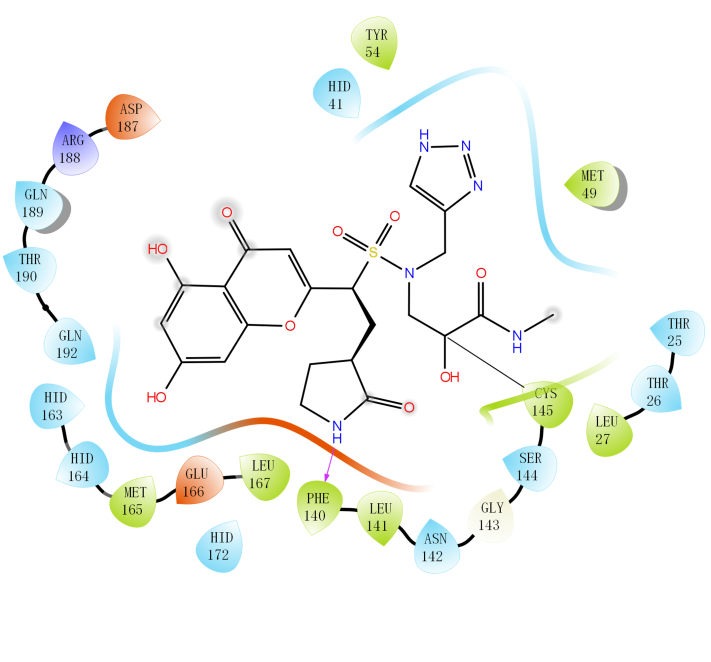

Figure 4. The covalent binding models of compound 46-14-1 and 46-14-2 in complex with the SARS-CoV-2 3CL pro. A: The covalent docking model between compound 46-14-1 (green sticks) with 3CL protease (yellow-orange surface). The oxyanion hole formed by the segment of $\alpha$ ketoamides is shown in the red circle. B: The detailed view of the interactions between the compound 46-14-1 and 3CL pro . C: The covalent docking model between compound 46-14-2 with 3CL protease. The molecule 46-14-2 is shown as green sticks and the protein is shown as brown surface. D: The 2D view of the interactions between the compound 46-14-1 and $3 \mathrm{CL}^{\text {pro }}$. 
A

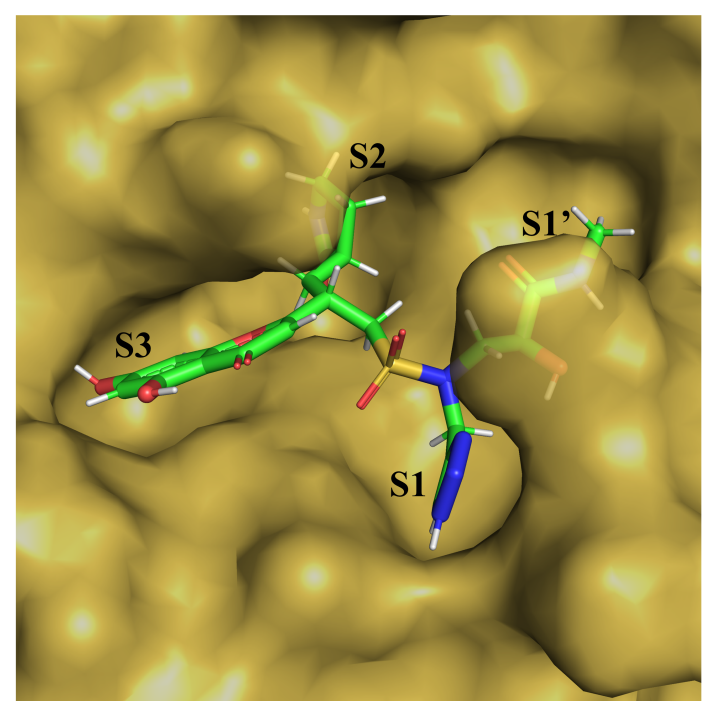

B

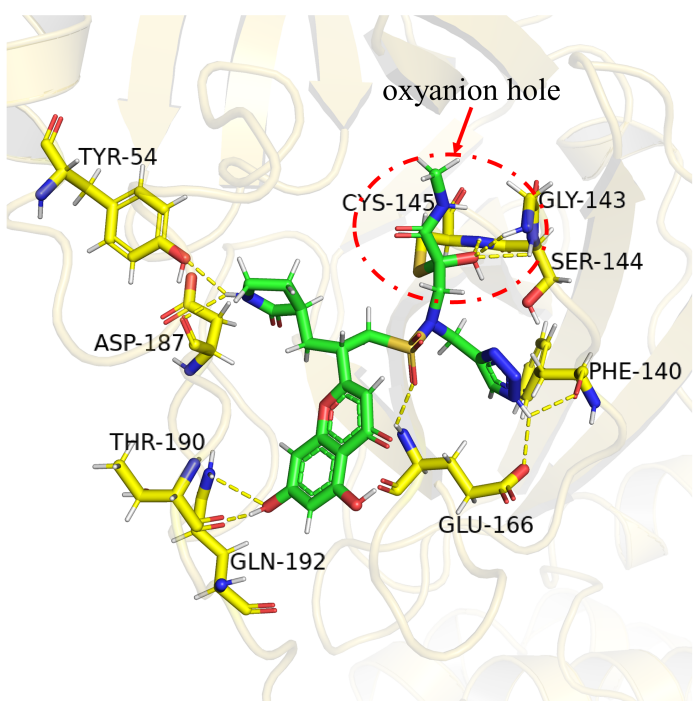

Figure 5. The covalent binding model of Compound 46-14-3 in SARS-CoV-2 3CL ${ }^{\text {pro }}$. A: Surface representation of SARS-CoV-2 3CL ${ }^{\text {pro }}$ (brown) complexed with 46-14-3 (green sticks). B: A stereo view showing 43-14-3 bound into the substrate-binding pocket of the SARS-CoV-2 $3 \mathrm{CL}^{\text {pro }}$ at $4 \AA$. The molecule 43-14-3 is shown as green sticks. Residues forming the H-bond are shown as yellow sticks. And the yellow dashes represent the H-bonds, as well as the oxyanion hole, is in the red circle region.<smiles>CC(CC1CCNC1=O)[C@H](C(=O)N(c1c[nH]nn1)C(C)/C=C/C=O)c1cc(=O)c2c(O)cc(O)cc2o1</smiles>

lead molecule 46<smiles>CNC(=O)C(=O)N(Cc1c[nH]nn1)S(=O)(=O)[C@H](C[C@@H]1CCNC1=O)c1cc(=O)c2c(O)cc(O)cc2o1</smiles>

molecule 46-14-2<smiles>CNC(=O)C(=O)CN(Cc1c[nH]nn1)C(=O)[C@H](C[C@@H]1CCNC1=O)c1cc(=O)c2c(O)cc(O)cc2o1</smiles>

molecule 46-14-1<smiles>CNC(=O)C(=O)CN(Cc1c[nH]nn1)S(=O)(=O)C[C@H](C[C@@H]1CCNC1=O)c1cc(=O)c2c(O)cc(O)cc2o1</smiles>

molecule 46-14-3

Figure 6. Structures of optimized compounds. 
The representatives were selected (46-14-1, 46-14-2 and 46-14-3 as Figure 6 displays), which may be further evaluated by molecular dynamics simulation to get the binding free energy and by quantum chemical calculation to get the reaction energy barrier. Meanwhile, 46-14-1, 46-14-2 and 46-14-3 are chosen as our candidates for chemical synthesis and anti-SARS-CoV-2 activity testing, which is ongoing.

\section{Discussions}

Computational approaches are particularly important for emerging diseases given the urgent need to provide timely solutions. In this work, our robust and efficient computational method and pipeline for designing compounds can provide useful drug candidates for treating SARS-CoV-2 infections. For more information about our AI model generated leads and SBDD optimized derivatives, please go to the library https://github.com/tbwxmu/2019-nCov. These candidates or their variants have a good chance to produce valid leads of anti-COVID-19 drugs. It is understood that the computational design requires experimental validations. While we are exploring experimental validations ourselves, we like to release these candidates promptly for other researchers to accelerate the development of anti-COVID-19 drugs given the emergency of seeking treatments for the disease.

Comparing with other deep RL methods, ADQN-FBDD has several highlights: 1) It directly modifies and generate molecular structures without format conversion problems while some other tools such as Insilico Medicine's GENTRL (https://insilico.com) may generate invalid SMILES output. 2) Most of the generative models require pre-training on a specific dataset and then produce the molecules with high similarities to a given training set. For example, the best molecule from Insilico Medicine for their target DDR1 was actually very similar to the kinase inhibitor Iclusig (ponatinib) on the market. ${ }^{37}$ ADQN-FBDD does not need pre-training at all and has a capacity to generate novel molecules. 3) The process of generating molecules is very 
efficient and effective as ADQN-FBBD is molecular fragment-based growing with the knowledge of chemical reactions, while other models are all atom-based with no rules of chemical reactions at all ${ }^{34,38,39}$.4) ADQN-FBDD is highly flexible and user-friendly for the medicinal chemists, who can easily inject their drug discovery experience into the reward function to guide the novel molecule generation. Our ADQN-FBDD and related pipeline can be used not only for designing anti-COVID-19 drugs but also other structure-based drug discoveries, especially for emerging infetious diseases that require treatments timely.

\section{Methods}

\section{Markov Decision Process for Molecule Generation}

Intuitively, the problem of chemical structure graph generation is formulated as learning a reinforced agent, which performs discrete actions of chemical reaction-based fragment addition or removal in a chemistry-aware Markov Decision Process (MDP). Formally, the components of MDP) include $M=\{S, A, P, R, \gamma\}$, where each term is defined as follows:

$S=\left\{S_{t}\right\}$ denotes the state space containing all possible intermediate and final generated molecular graphs. Each $s_{t}$ is a tuple of $(s, t) . s$ stands for a valid molecule structure and $t$ is the time step. For the initial state $s_{0}$, the structure can be represented as a specific core as Figure $2 \mathrm{C}$ indicates or randomly chosen from the prepared fragment library at time $t=0$. We also limit the maximum number of time steps $T$ in our molecule fragment based MDP, which defines the set of terminal states as $\left\{s_{t} \mid t=T\right\}$ containing all the states with the number of steps reaching the maximum allowed value $T$.

$\mathrm{Ac}=\left\{A_{t}\right\}$ denotes a set of actions that describe the modification made on the current molecule structure at each time step $t$. Here, each action can be classified into three 
categories: fragment addition, fragment deletion, and no modification.

$P=p\left(s_{t+1} \mid s_{t} \ldots s_{0}\right)=p\left(s_{t+1} \mid s_{t}, a_{t}\right)$ is the basic assumption in MDP. The state transition probability, which specifies the next possible state given the current state and action at time step t. Here, we define the state transition to be deterministic. For example, as Figure $2 \mathrm{C}$ indicates $\mathrm{S}_{0}$ to $\mathrm{S}_{1}$, by adding a $1 H-1,2,3$-triazol-4-yl fragment on $S_{0}$, the next state $S_{l}$ will be the new structure of added $1 H-1,2,3-$ triazol-4-yl with a probability of 1 .

$R$ is the reward function that specifies the reward after reaching state $S_{t}, \gamma \in(0,1]$ is the discount factor and typically $\gamma=0.9$ in our study. In our framework, the state always has a valid and complete chemical structure at each step as Figure $2 \mathrm{C}$ indicates. A reward is given not just at the terminal states, but at each step. Both intermediate rewards and final rewards are used to guide the behavior of the reinforcement learning (RL) agent. So, there is no delayed or sparse reward issue as many other reinforced frameworks suffered. ${ }^{40,41}$ Furthermore, to ensure that the last state is rewarded the most, we use $\gamma^{T-t}$ to discount the value of the rewards at state $s_{t}$. In addition, our reward function can directly integrate the experience of medicinal chemists. For example, given a core of interest, medicinal chemists may add biased fragments of their interests and their input can be used to design a reward function that gives high reward signal values to biased fragments so that ADQN-FBDD may have a better chance to generate the desired structures.

\section{Chemical Environment Design}

In our RL framework, the chemical environment receives action $a_{\mathrm{t}}$ from the agent and emits scalar reward $r_{t}$ and state $s_{t}^{\prime}$ to the agent as Figure $2 \mathrm{~A}$ shows. Note that the definition of the environment state is different from the general approach that the environment state is only the environment's private representation invisible to the agent. We define the state of the chemical environment $s_{t}^{\prime}$ as the intermediate generated molecule structure at time step t, which is fully observable by the RL agent. Simply, 
the environment's state $s_{t}^{\prime}$ is the agent's state $s_{t+1}$. For the task of molecule generation, the environment incorporates rules of chemistry. In our study, chemistry rules are not only the basic chemical valency, but also the rules about adding and removing the fragments derived from known inhibitors based on chemical reactions. The detail information of 45 defined chemical reaction rules is presented in Table S2.

\section{Agent Design}

As indicated in Figure 2A, the basic model of our ADQN-FBDD is an advanced Qnetwork. The goal of molecule generation is equally to fit a $\mathrm{Q}$ function $Q\left(s_{t}, a_{t}\right)$ to make the agent choose the action $a_{t}$ at state $s_{t}$ that maximizes the future expected $\gamma$-discounted cumulative rewards with policy $\pi$. Mathematically, given the agent's policy $\pi$, the value of the state-action pair $\mathrm{Q}^{\pi}\left(s_{t}, a_{t}\right)$ and the value of state $\mathrm{V}^{\pi}\left(s_{t}\right)$ are defined as, respectively:

$$
\begin{aligned}
& Q^{\pi}\left(s_{t}, a_{t}\right)=E_{a_{t} \sim \pi\left(s_{t}\right)}\left[\sum_{n=t}^{T} \gamma^{T-n} \cdot \mathrm{R}\left(s_{n}, a_{n}\right)\right] \\
& \quad=E_{a_{t} \sim \pi\left(s_{t}\right)}\left[\mathrm{R}\left(s_{t}, a_{t}\right)+\gamma \cdot E_{a_{t+1} \sim \pi\left(s_{t+1}\right)}\left(Q^{\pi}\left(s_{t+1}, a_{t+1}\right)\right]\right. \\
& V^{\pi}\left(s_{t}\right)=E_{a_{t} \sim \pi\left(s_{t}\right)}\left[Q^{\pi}\left(s_{t}, a_{t}\right)\right]
\end{aligned}
$$

where $E_{a_{t} \sim \pi\left(s_{t}\right)}$ is the expectation within policy $\pi$ on state $s_{t}$ taken $a_{t}$ and $\mathrm{R}\left(s_{n}, a_{n}\right)$ denotes the reward at step $n$. Value function $\mathrm{Q}^{\pi}\left(s_{t}, a_{t}\right)$ measures the value of taking action $a_{t}$ on state $s_{t} . \mathrm{V}^{\pi}\left(s_{t}\right)$ is the value of being at state $s_{t}$ means how good to be in this state. Obviously, $\mathrm{V}^{\pi}\left(\mathrm{s}_{\mathrm{t}}\right)$ can be seen as a part of $\mathrm{Q}^{\pi}\left(s_{t}, a_{t}\right)$. Then, the rest part from $\mathrm{Q}^{\pi}\left(s_{t}\right.$, $\left.a_{t}\right)$ can be defined as the so-called advantage function $\mathrm{A}^{\pi}\left(s_{t}, a_{t}\right)^{42}$ as:

$$
\mathrm{A}^{\pi}\left(s_{t}, a_{t}\right)=Q^{\pi}\left(s_{t}, a_{t}\right)-V^{\pi}\left(s_{t}\right)
$$

Intuitively, the advantage value shows how advantageous selecting the action is relative to the others at the same given state. Then Eqn. (2) can be rewritten according to Eqn. (4): 
$V^{\pi}\left(s_{t}\right)=E_{a_{t} \sim \pi\left(s_{t}\right)}\left[V^{\pi}\left(s_{t}\right)+\mathrm{A}^{\pi}\left(s_{t}, a_{t}\right)\right]=V^{\pi}\left(s_{t}\right)+E_{a_{t} \sim \pi\left(s_{t}\right)}\left[\mathrm{A}^{\pi}\left(s_{t}, a_{t}\right)\right]$

Obviously, $E_{a_{t} \sim \pi\left(s_{t}\right)}\left[\mathrm{A}^{\pi}\left(s_{t}, a_{t}\right)\right]=0$. To avoid the issue of identifiability, we deduct the mean value from the prediction and the Q-function of dueling DQN can be defined as:

$Q^{\pi}\left(s_{t}, a_{t} ; \Theta, \alpha, \beta\right)=V^{\pi}\left(s_{t} ; \Theta, \beta\right)+\left(\mathrm{A}^{\pi}\left(s_{t}, a_{t} ; \Theta, \alpha\right)-\frac{1}{|\mathrm{Ac}|} \sum_{a_{t}^{\prime}} \mathrm{A}^{\pi}\left(s_{t}, a_{t}^{\prime} ; \Theta, \alpha\right)\right)$

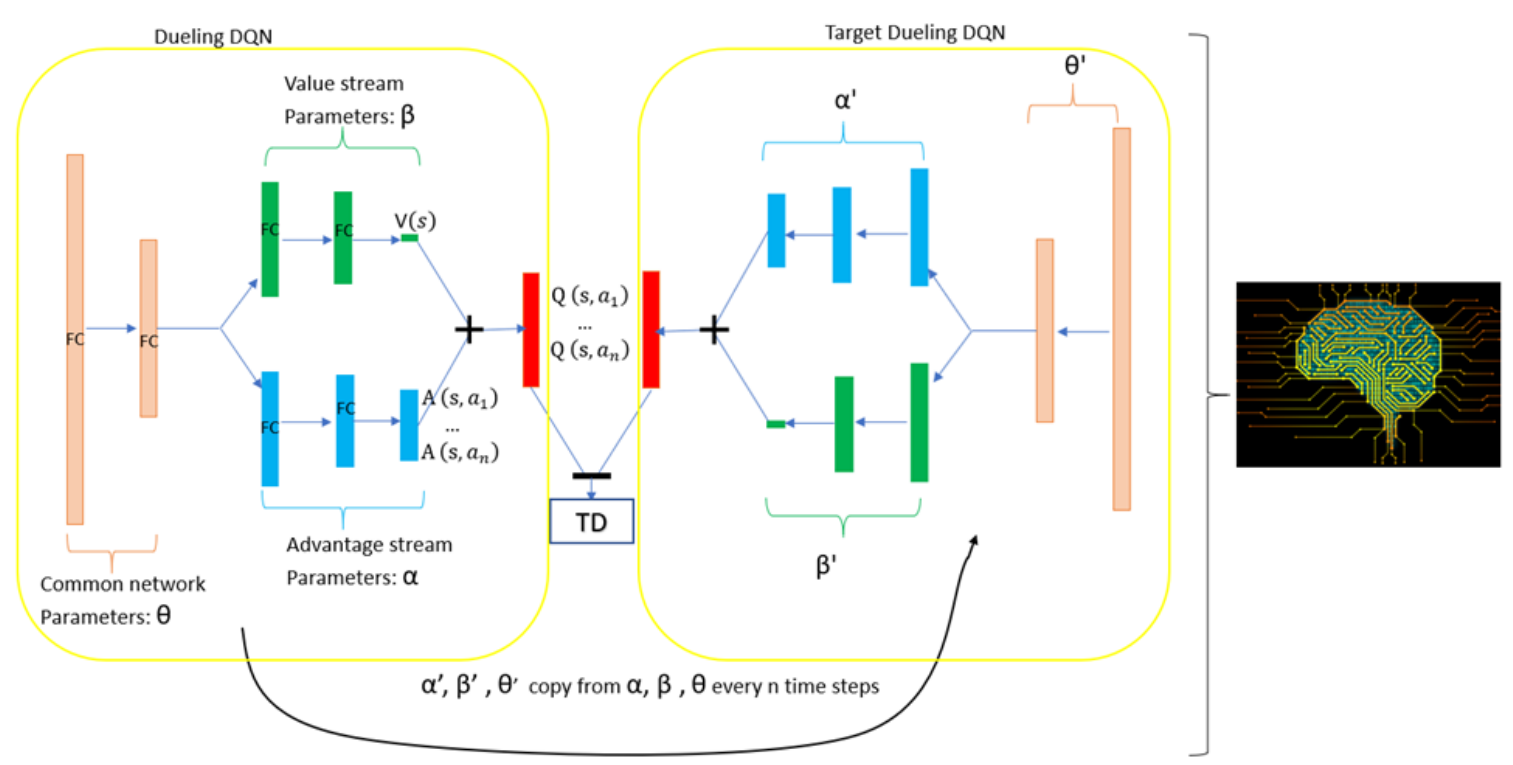

Figure 7. Architecture of used advance deep Q-learning network (ADQN). TD is the temporal difference error.

Note that $\Theta, \alpha$ and $\beta$ come from the dueling Q-network as Figure 7 indicates. $|\mathrm{Ac}|$ is the size of action space and $a_{t}^{\prime} \epsilon A c$. To make our RL agent more robust to be more stable learning and to handle the problem of the overestimation of Q-values, double Qnetwork $^{43}$ and fixed Q-targets ${ }^{44}$ are also incorporated:

$T D=Q^{\pi}\left(s_{t}, a_{t} ; \Theta, \alpha, \beta\right)-\left[\mathrm{R}\left(s_{t}, a_{t}\right)+\gamma\right.$.

$\left.Q_{t a r}^{\pi}\left(s_{t+1}, \operatorname{argmax}_{a_{t+1}} Q^{\pi}\left(s_{t+1}, a_{t+1} ; \Theta, \alpha, \beta\right) ; \Theta^{\prime}, \alpha^{\prime}, \beta^{\prime}\right)\right]$

where, TD is the temporal-difference; $Q_{\text {tar }}^{\pi}$ is another dueling DQN network as the target network and its parameters $\left(\Theta^{\prime}, \alpha^{\prime}, \beta^{\prime}\right)$ keep fixed and copy from the dueling DQN 
$Q^{\pi}$ every $m$ steps ( $m=20$ as we used). To update the parameters $(\Theta, \alpha, \beta)$ from the dueling DQN as Figure 7 displays, we can train our RL agent by minimizing the loss function:

$$
l(\Theta, \alpha, \beta)=E\left[f_{l}(T D)\right]
$$

where $\boldsymbol{E}$ is the expectation. As the L2 loss has a disadvantage of the tendency to be dominated by outliers, we use the Huber loss as the loss function $f_{l}$ :

$$
f_{l}(x)= \begin{cases}|x|-0.5 & \text { if }|x| \geq 1 \\ 0.5 * x^{2} & \text { if }|x|<1\end{cases}
$$

\section{Prioritized Experience Replay}

Prioritized Experience Replay (PER) ${ }^{45}$ is a technique to enable reinforcement learning agents remember and reuse experience from the past, and to replay important transitions more frequently. PER is very useful for replaying some less frequent experiences. Here, we use the same code of "Prioritized Replay Buffer" from open AI's gym with version 0.15.4. ${ }^{46}$ Finally, our RL agent is the double dueling deep Q learning with fixed qtargets and prioritized experience replay.

\section{Fragment Library Design}

The fragment-based approach to drug discovery (FBDD) has been established as an efficient tool in the search for new drugs. ${ }^{47}$ The idea of FBDD is that proper optimization of each unique interaction in the binding site and subsequent incorporation into a single molecular entity should produce a compound with a binding affinity that is the sum of the individual interactions. However, the widely used fragment libraries only consider the diversity of fragments, such as the ZINC fragment database. They have a very low probability to exhibit the desired bioactivity for a given protein. 
To combine the idea of FBDD with our RL framework, we first collected and built a SARS-CoV-2 3CL pro inhibitor dataset containing 284 reported molecules. Then using the improved BRICS algorithm ${ }^{48}$ to split those molecules to get the fragment library target on SARS-CoV-2 3CL ${ }^{\text {pro }}$ as the flowchart displayed in Figure 1's yellow box. An elaborate filtering cascade accompanied by manual inspection and the rules can be changed based on the needs in different studies. Our fragment library contains 316 fragments with molecular weight $<200$ daltons, the minimum number non-hydrogen atom $>1$ and the maximum $\leq 25$. The fragments directly from the existing inhibitors based on the chemical retrosynthetic rules are always true substructures and may have a high quality of bioactivity targeting $3 \mathrm{CL}^{\text {pro }}$. It is worth noting that the quality of the designed fragment library directly affects the behavior of the chemical environment of ADQN-FBDD.<smiles>Cc1cc(C(=O)N[C@@H](C)C(=O)NC(C(=O)N[C@@H](CC(C)C)C(=O)N[C@@H](/C=C/C(=O)OCc2ccccc2)C[C@H]2CCNC2=O)C(C)C)no1</smiles><smiles>C/C=C/C(C)N</smiles>

$(E)$-4-aminopent-2-enal<smiles>O=C(/C=C/c1ccccc1)NC(=O)C(CC1CC1)NC(=O)C(=O)NCc1ccccc1</smiles><smiles>CC(N)C(=O)C=O</smiles>

3-amino-2-oxobutanal

Figure 8. Structures of the chosen cores

\section{Core Selection}

Studies have identified various scaffolds or core structures that have privileged characteristics in terms of the activity of a certain target. ${ }^{49,50,51}$ Core structure selection is the starting point in a scaffold-based drug discovery. Choosing or designing a proper 
initial scaffold is never trivial, and medicinal chemists may need enough experience to get such a skill. Luckily, there are serval reported privileged core structures targeting SARS M ${ }^{\text {pro }}$ 3, 52 Here, we chose 4-aminopent-2-enal and 3-amino-2-oxobutanal as the starting cores as Figure 8 displays, because both cores have been validated to generate covalent bonds with the Cys145 of SARS or SARS-CoV-2 3CL pro.

\section{Reward Design}

Most reported RL methods using the complete structure information of a positive drug or inhibitor as the template, ${ }^{38,39}$ and they design a reward function for the RL agent to learn to regenerate the template structure or generate highly similar structures to the template. This way may be useful in testing the performance of RL methods but not suitable in a real-world drug design because no one knows the complete structural information of the novel molecule. A more practical approach is to learn the structure features of existing drugs or inhibitors to local, focused chemical space for a specific protein target. Instead of simply putting attention on the diversity of molecule structure, we explore the possibility of generating novel molecules based on the existing knowledge. Here, we designed a deep reinforcement learning reward (DRL-reward) function that consists of the final property score, containing specific fragments (CSF) score and pharmacophore score as:

$$
\begin{aligned}
& R(s)=w_{\text {pro }} \cdot f_{\text {pro }}(s)+w_{\text {con }} \cdot f_{\text {con }}(s)+w_{\text {pha }} \cdot f_{\text {pha }}(s) \\
& f_{\text {pro }}(s)= \begin{cases}1 & \text { if } Q E D(S)>0.1 \\
0 & \text { else }\end{cases}
\end{aligned}
$$

where $w_{\text {pro }}$ represents weight for the quantitative estimate of drug-likeness (QED) property and its default value is 0.1 . $f_{\text {pro }}$ stands for the QED. QED values can range from 0 (all properties are unfavourable) to 1 (all properties are favourable), which are calculated by eight molecular properties. ${ }^{53}$ The score function $f_{c s f}$ of containing specific fragments (csf) is: 


$$
\begin{aligned}
& f_{C S F}(s)=\left\{\begin{array}{l}
F_{c s f}(s) \text { if } F_{C S F}(s)>0.9 \text { else } 0 \\
0 \text { else }
\end{array}\right. \\
& F_{C S F}(s)=\frac{n_{\text {match }}}{N_{\text {total }}}
\end{aligned}
$$

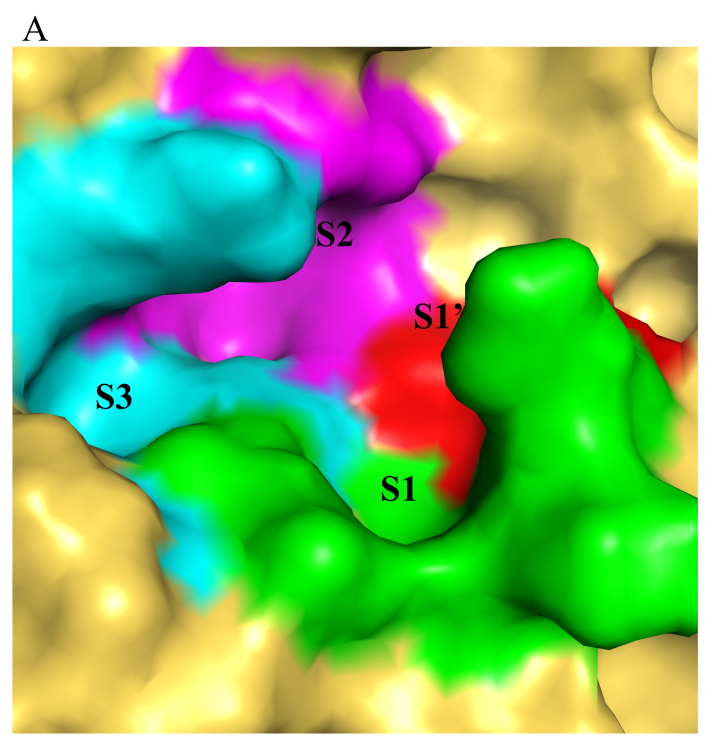

B

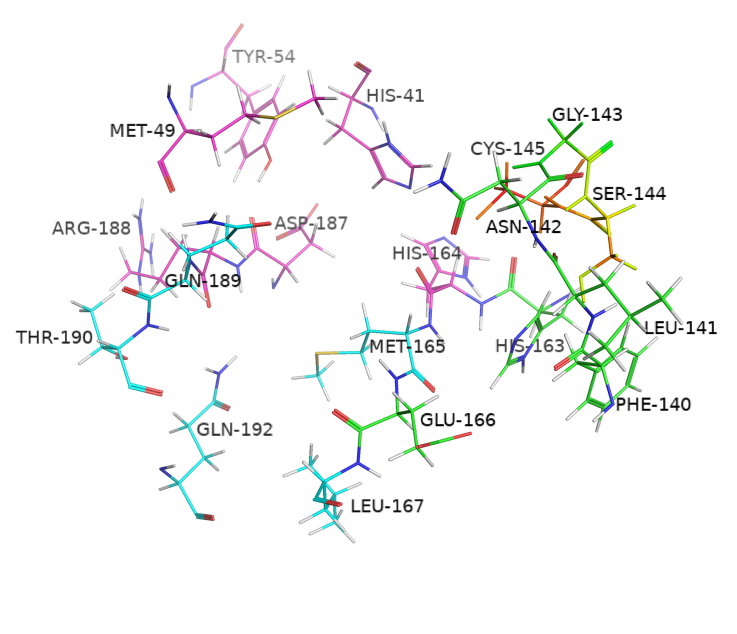

Figure 9. Binding site of SARS-CoV-2 $3 \mathrm{CL}^{\text {pro }}$. A: The subsites that complement the substrate-binding pocket are shown as surface, which are assigned as S1 (green), S1' (red), S2 (magenta), and S3(cyan). B: The key residues of the binding site are displayed by green, red, magenta and cyan lines ( $\mathrm{S} 1, \mathrm{~S} 1$ ', S2 and S3). The pictures of the binding site are generated using PyMol ((http://www.pymol.org/).

The binding site of SARS-CoV-2 3CL pro (Figure 9) is commonly divided into the catalytic activity center (His41 and Cys145, specified as S1') and several subsites, defined as S1 (His163, Glu166, Phe140, Leu141 and Asn142), S1' (His41, Cys145, Gly143 and Ser144), S2 (Tyr54, Asp187, His41, Arg188, His164 and Met49), and S3 (Thr190, Gln192, Glu166, Met165, Leu167 and Gln189). Each subsite may have its own favorable binding fragment. When generated structures (including the intermediates) containing those favorable fragments, $f_{c s f}$ is equivalent to give an additional reward to our RL agent. $w_{\text {con }}$ controls the contribution of the biased fragment to the reward signal and the default value is 0.6. $n_{\text {match }}$ stands for how many biased fragments have been matched in one generated structure. $N_{\text {total }}$ is the number of biased fragments defined based on our knowledge learned from related work. $f_{p h a}$ 
represents the score function of pharmacophores, which mainly depends on the ligandprotein interaction mode from the crystal structure (PDB ID: 6LU7):

$f_{p h a}(s)= \begin{cases}1 & \text { if matchs the defined pharmacophores } \\ 0 & \text { else }\end{cases}$

The pharmacophores plot was added in Figure S5. $w_{p h a}$ controls the contribution of the pharmacophore score to the reward and the default value is 0.4 .

\section{Molecular Generation and Selection}

As discussed in the above reward design part, our reward function considers the molecular descriptor thresholds $(\mathrm{QED}>0.1)$, the defined pharmacophores and biased fragments. In total, 4,922 unique valid structures were automatically generated and all matched the defined rules by using ADQN-FBDD without any pre-training as many other methods need. ${ }^{35,40,41,54,55}$ Next, All the molecules with high deep reinforcement learning scores (DRL score: $R(S)>0.6$ ) were kept (47 molecules). Then, these 47 unique molecules were prepared to generate at least 1 conformation with the local energy minimization using the OPLS-2005 force field by the "ligand prepare" module of Schrödinger 2015 software. The 47 unique molecules generated a total of 163 3D conformations before docking into the substrate-binding site of SARS-CoV-2 3CL ${ }^{\text {pro }}$. Considering the balance between precision and calculation time, the stand-precision (SP) Glide ${ }^{56}$ was firstly used to predict the possible non-covalent binding poses in this binding site and the binding site grid centered on the original ligand N3 $3^{57}$ with $20 \AA$ buffer dimensions. Following this non-covalent docking, we also calculated the covalent docking poses and scores for those 47 molecules. We reordered the docking results mainly based on the covalent docking score and the RMSD value from covalent docking pose to non-covalent (Table S1).

\section{Supplementary information}


Supplementary dataset and additional information of this paper can be found at https://github.com/tbwxmu/2019-nCov.

\section{Acknowledgements}

We would like to thank Prof. Dongqing Wei's group and the PCL lab for their generous support of high-performance computing resources. BT's work was funded by the program of China Scholarships Council No. 201806310017. DX's effort was supported by the US National Institutes of Health grant R35-GM126985.

\section{Authors' contributions}

BT and DX designed the study. BT developed the AI-aided methods and wrote the manuscript. All authors contributed to the interpretation of results. All authors reviewed and edited the manuscript. All authors read and approved the final manuscript.

\section{Data and Code availability}

The code for the ADQN-FBDD and related data in this paper will be available at https://github.com/tbwxmu/2019-nCov upon acceptance of this paper for journal publication.

\section{Competing interests}

The authors declare that they have no competing interests.

\section{References:}

1. Gorbalenya AE. Severe acute respiratory syndrome-related coronavirus-The species and its viruses, a statement of the Coronavirus Study Group. BioRxiv, (2020).

2. Coronavirus COVID-19 Global Cases by Johns Hopkins CSSE, available from : https://forum.truckersmp.com/index.php?/topic/93003-coronavirus-covid-19-global-cases-by- 
johns-hopkins-csse $/ \&$ tab=comments.

3. Zhang L, et al. Alpha-ketoamides as broad-spectrum inhibitors of coronavirus and enterovirus replication Structure-based design, synthesis, and activity assessment. Journal of Medicinal Chemistry, (2020).

4. 卢洪洲 陈凌席刘李李尚王沈. 洛匹那韦利托那韦和阿比多尔用于治疗新型冠状病毒肺 炎的有效性研究. 中华传染病杂志 38, (2020).

5. Liu X, Wang X-J. Potential inhibitors for 2019-nCoV coronavirus M protease from clinically approved medicines. bioRxiv, (2020).

6. Yang $\mathrm{H}$, et al. Design of wide-spectrum inhibitors targeting coronavirus main proteases. PLoS biology 3, (2005).

7. Singh J, Petter RC, Baillie TA, Whitty A. The resurgence of covalent drugs. Nature reviews Drug discovery 10, 307-317 (2011).

8. Tuley A, Fast W. The taxonomy of covalent inhibitors. Biochemistry 57, 3326-3337 (2018).

9. Jain RP, et al. Synthesis and evaluation of keto-glutamine analogues as potent inhibitors of severe acute respiratory syndrome 3CLpro. Journal of medicinal chemistry 47, 6113-6116 (2004).

10. Wu C-Y, et al. Small molecules targeting severe acute respiratory syndrome human coronavirus. Proceedings of the National Academy of Sciences 101, 10012-10017 (2004).

11. Ghosh AK, et al. Design and synthesis of peptidomimetic severe acute respiratory syndrome chymotrypsin-like protease inhibitors. Journal of medicinal chemistry 48, 6767-6771 (2005).

12. Shie J-J, et al. Discovery of potent anilide inhibitors against the severe acute respiratory syndrome 3CL protease. Journal of medicinal chemistry 48, 4469-4473 (2005).

13. Shie J-J, et al. Inhibition of the severe acute respiratory syndrome $3 \mathrm{CL}$ protease by peptidomimetic $\alpha$, $\beta$-unsaturated esters. Bioorganic \& medicinal chemistry 13, 5240-5252 (2005).

14. Al-Gharabli SI, et al. An efficient method for the synthesis of peptide aldehyde libraries employed in the discovery of reversible SARS coronavirus main protease (SARS-CoV Mpro) inhibitors. ChemBioChem 7, 1048-1055 (2006).

15. Lu I-L, et al. Structure-based drug design and structural biology study of novel nonpeptide inhibitors of severe acute respiratory syndrome coronavirus main protease. Journal of medicinal chemistry 49, 5154-5161 (2006). 
16. Tsai K-C, et al. Discovery of a novel family of SARS-CoV protease inhibitors by virtual screening and 3D-QSAR studies. Journal of medicinal chemistry 49, 3485-3495 (2006).

17. Wu C-Y, et al. Stable benzotriazole esters as mechanism-based inactivators of the severe acute respiratory syndrome 3CL protease. Chemistry \& biology 13, 261-268 (2006).

18. Akaji K, Konno H, Onozuka M, Makino A, Saito H, Nosaka K. Evaluation of peptide-aldehyde inhibitors using R188I mutant of SARS 3CL protease as a proteolysis-resistant mutant. Bioorganic \& medicinal chemistry 16, 9400-9408 (2008).

19. Ghosh AK, et al. Design, synthesis and antiviral efficacy of a series of potent chloropyridyl ester-derived SARS-CoV 3CLpro inhibitors. Bioorganic \& medicinal chemistry letters $\mathbf{1 8}$, 5684-5688 (2008).

20. Shao Y-M, et al. Design, synthesis, and evaluation of trifluoromethyl ketones as inhibitors of SARS-CoV 3CL protease. Bioorganic \& medicinal chemistry 16, 4652-4660 (2008).

21. Kuo C-J, et al. Individual and common inhibitors of coronavirus and picornavirus main proteases. FEBS letters 583, 549-555 (2009).

22. Ramajayam R, Tan K-P, Liu H-G, Liang P-H. Synthesis and evaluation of pyrazolone compounds as SARS-coronavirus 3C-like protease inhibitors. Bioorganic \& medicinal chemistry 18, 7849-7854 (2010).

23. Ryu YB, et al. Biflavonoids from Torreya nucifera displaying SARS-CoV 3CLpro inhibition. Bioorganic \& medicinal chemistry 18, 7940-7947 (2010).

24. Akaji K, et al. Structure-based design, synthesis, and evaluation of peptide-mimetic SARS 3CL protease inhibitors. Journal of medicinal chemistry 54, 7962-7973 (2011).

25. Jacobs J, et al. Discovery, synthesis, and structure-based optimization of a series of N-(tertbutyl)-2-(N-arylamido)-2-(pyridin-3-yl) acetamides (ML188) as potent noncovalent small molecule inhibitors of the severe acute respiratory syndrome coronavirus (SARS-CoV) $3 \mathrm{CL}$ protease. Journal of medicinal chemistry 56, 534-546 (2013).

26. Ren Z, et al. The newly emerged SARS-like coronavirus HCoV-EMC also has an" Achilles' heel": current effective inhibitor targeting a 3C-like protease. Protein \& cell 4, 248 (2013).

27. Thanigaimalai $\mathrm{P}$, et al. Development of potent dipeptide-type SARS-CoV 3CL protease inhibitors with novel P3 scaffolds: Design, synthesis, biological evaluation, and docking studies. European journal of medicinal chemistry 68, 372-384 (2013).

28. Turlington M, et al. Discovery of N-(benzo [1, 2, 3] triazol-1-yl)-N-(benzyl) acetamido) phenyl) 
carboxamides as severe acute respiratory syndrome coronavirus (SARS-CoV) 3CLpro inhibitors: identification of ML300 and noncovalent nanomolar inhibitors with an induced-fit binding. Bioorganic \& medicinal chemistry letters 23, 6172-6177 (2013).

29. Kumar V, et al. Identification and evaluation of potent Middle East respiratory syndrome coronavirus (MERS-CoV) 3CLPro inhibitors. Antiviral research 141, 101-106 (2017).

30. Tang B, Kramer ST, Fang M, Qiu Y, Wu Z, Xu D. A self-attention based message passing neural network for predicting molecular lipophilicity and aqueous solubility. Journal of Cheminformatics 12, 1-9 (2020).

31. Liu K, et al. Chemi-Net: a molecular graph convolutional network for accurate drug property prediction. International journal of molecular sciences 20, 3389 (2019).

32. Wang X, Li Z, Jiang M, Wang S, Zhang S, Wei Z. Molecule Property Prediction Based on Spatial Graph Embedding. Journal of chemical information and modeling 59, 3817-3828 (2019).

33. Wu Z, et al. MoleculeNet: a benchmark for molecular machine learning. Chemical science $\mathbf{9}$, 513-530 (2018).

34. Jin W, Barzilay R, Jaakkola T. Junction tree variational autoencoder for molecular graph generation. arXiv preprint arXiv:180204364, (2018).

35. You J, Liu B, Ying Z, Pande V, Leskovec J. Graph convolutional policy network for goaldirected molecular graph generation. In: Advances in neural information processing systems (2018).

36. Zhu K, et al. Docking Covalent Inhibitors: A Parameter Free Approach To Pose Prediction and Scoring. Journal of Chemical Information \& Modeling 54, 1932-1940 (2014).

37. Walters WP, Murcko M. Assessing the impact of generative AI on medicinal chemistry. Nature Biotechnology, 1-3 (2020).

38. Olivecrona M, Blaschke T, Engkvist O, Chen H. Molecular de-novo design through deep reinforcement learning. Journal of cheminformatics $\mathbf{9}, 48$ (2017).

39. Zhou Z, Kearnes S, Li L, Zare RN, Riley P. Optimization of molecules via deep reinforcement learning. Scientific reports 9, 1-10 (2019).

40. Popova M, Isayev O, Tropsha A. Deep reinforcement learning for de novo drug design. Science advances 4, eaap7885 (2018).

41. Zhavoronkov A, et al. Deep learning enables rapid identification of potent DDR1 kinase inhibitors. Nature biotechnology 37, 1038-1040 (2019). 
42. Wang Z, Schaul T, Hessel M, Van Hasselt H, Lanctot M, De Freitas N. Dueling network architectures for deep reinforcement learning. arXiv preprint arXiv:151106581, (2015).

43. Van Hasselt H, Guez A, Silver D. Deep reinforcement learning with double q-learning. In: Thirtieth AAAI conference on artificial intelligence (2016).

44. Simonini T. Improvements in Deep Q Learning: Dueling Double DQN, Prioritized Experience Replay, and fixed Q-targets. Červenec (2018).

45. Schaul T, Quan J, Antonoglou I, Silver D. Prioritized experience replay. arXiv preprint arXiv:151105952, (2015).

46. Brockman G, et al. Openai gym. arXiv preprint arXiv:160601540, (2016).

47. Speck-Planche A. Recent advances in fragment-based computational drug design: tackling simultaneous targets/biological effects. Future Science (2018).

48. Degen J, Wegscheid-Gerlach C, Zaliani A, Rarey M. On the Art of Compiling and Using'DrugLike'Chemical Fragment Spaces. ChemMedChem: Chemistry Enabling Drug Discovery 3, 1503-1507 (2008).

49. Varin T, Schuffenhauer A, Ertl P, Renner S. Mining for bioactive scaffolds with scaffold networks: improved compound set enrichment from primary screening data. Journal of chemical information and modeling 51, 1528-1538 (2011).

50. Schuffenhauer A, Ertl P, Roggo S, Wetzel S, Koch MA, Waldmann H. The scaffold treevisualization of the scaffold universe by hierarchical scaffold classification. Journal of chemical information and modeling 47, 47-58 (2007).

51. Reis J, Gaspar A, Milhazes N, Borges F. Chromone as a Privileged Scaffold in Drug Discovery: Recent Advances: Miniperspective. Journal of medicinal chemistry 60, 7941-7957 (2017).

52. Pillaiyar T, Manickam M, Namasivayam V, Hayashi Y, Jung S-H. An Overview of Severe Acute Respiratory Syndrome-Coronavirus (SARS-CoV) 3CL Protease Inhibitors: Peptidomimetics and Small Molecule Chemotherapy. Journal of Medicinal Chemistry, (2016).

53. Bickerton GR, Paolini GV, Besnard J, Muresan S, Hopkins AL. Quantifying the chemical beauty of drugs. Nature chemistry 4, 90 (2012).

54. Elton DC, Boukouvalas Z, Fuge MD, Chung PW. Deep learning for molecular design — a review of the state of the art. Molecular Systems Design \& Engineering 4, 828-849 (2019).

55. Yang X, Wang Y, Byrne R, Schneider G, Yang S. Concepts of artificial intelligence for 
computer-assisted drug discovery. Chemical reviews 119, 10520-10594 (2019).

56. Friesner RA, et al. Extra precision glide: Docking and scoring incorporating a model of hydrophobic enclosure for protein- ligand complexes. Journal of medicinal chemistry 49, 61776196 (2006).

57. Jin Z, et al. Structure-based drug design, virtual screening and high-throughput screening rapidly identify antiviral leads targeting COVID-19. bioRxiv, 2020.2002.2026.964882 (2020). 